\title{
The interaction of the projectile with moving plates and rods
}

\author{
A. Gerasimov and S. Pashkov
}

Tomsk State University, Research Institute of Applied Mathematics and Mechanics, 634050 Tomsk, Russia

\begin{abstract}
The study of the problem of protecting the elements of constructions from impact loadings is very important due to the need of constant perfection of the means of shock-wave impact on the objects of modern technology. The problem of creating a reliable protective system dictates necessity of studying different ways to counteract to high-velocity elongated projectiles. The interaction of projectiles with plates and rods which are thrown towards by HE is investigated. The strain and fracture of the projectile sharply reduce its penetrating ability.
\end{abstract}

\section{The equations describing movement of compressed elastic-plastic body taking into account probabilistic fracture behavior}

The problem is solved in 3-D statement in view of natural heterogeneity of real materials structure affecting distribution of physical-mechanical characteristics along the volume of construction elements and being one of the factors, defining destruction character of the latter.

The necessity to account the given factor for equations of deformable solid mechanics dictates the application of probabilistic laws of physical-mechanical characteristics distribution along the volume of the construction under consideration.

The equations describing spatial adiabatic movement of the strong compressed solid are differential result of fundamental laws of conservation of mass, pulse and energy. In general they have the following form [1-3].

The equation of continuity

$$
\frac{1}{\rho} \frac{d \rho}{d t}+\frac{\partial v_{i}}{\partial x_{i}}=0
$$

The equations of movement

$$
\rho \frac{d v_{i}}{d t}=\rho F_{i}-\frac{\partial P}{\partial x_{i}}+\frac{\partial S_{i j}}{\partial x_{j}} .
$$

The equation of energy

$$
\rho \frac{d E}{d t}=S_{i j} \varepsilon_{i j}+\frac{P}{\rho} \frac{d \rho}{d t},
$$

where $x_{i}$ - coordinates; $t$ - time; $\rho_{0}$ - initial density; $\rho$ - current density; $v_{i}$ - velocity vector components; $F_{i}$ - components of the vector of mass forces; $S_{i j}$ - stress deviator components; $E$ - specific internal energy; $\varepsilon_{i j}-$ components of the strain rate tensor; $P$ - pressure.

Equations (1)-(3) must be added by equations that take into account the relevant thermodynamic effects related to the adiabatic compression of the medium and the strength of the medium. In general at action of forces on a solid deformable body both the volume (density) and shape of the body change, and at different dependences. Therefore the tensor of stress is represented as the sum of a pressure and the stress deviator components.

$$
\begin{aligned}
& \sigma_{i j}=S_{i j}-P \delta_{i j}, \quad i, j=1,2,3 \\
& \delta_{i j}=1, \quad i=j, \\
& \delta_{i j}=0, \quad i \neq j
\end{aligned}
$$

where $\delta_{i j}-$ Kronecker symbol.

To describe resistance of a solid to shear, we use the following ratios:

$$
\begin{gathered}
2 \mu\left(e_{i j}-\frac{1}{3} e_{k k} \delta_{i j}\right)=\frac{D S_{i j}}{D t}+\lambda S_{i j} \\
\frac{D S_{i j}}{D t}=\frac{d S_{i j}}{d t}-S_{i k} \omega_{j k}-S_{j k} \omega_{i k} \\
2 \omega_{i j}=\frac{\partial v_{i}}{\partial x_{j}}-\frac{\partial v_{j}}{\partial x_{i}} \\
2 e_{i j}=\frac{\partial v_{i}}{\partial x_{j}}+\frac{\partial v_{j}}{\partial x_{i}}
\end{gathered}
$$

and also plasticity condition

$$
J_{2}=\frac{1}{2} S_{i j} S_{i j}=\frac{1}{3} \sigma^{2},
$$

where $e_{i j}$ - strain rate tensor components; $\mu$ - shear modulus; $\sigma$ - dynamic yield stress; $D / D t$ - Jaumann derivative.

The equation of state was chosen in the form of Mi-Gruneisen

$$
P=\frac{K\left(1-G_{0} \xi / 2\right)}{(1-c \xi)^{2}} \xi+\rho_{0} G_{0} E
$$

where $G_{0}-$ Gruneisen coefficient; $c, K-$ material constants; $\rho_{0}-$ initial density; $\xi=1-\rho_{0} / \rho$. 


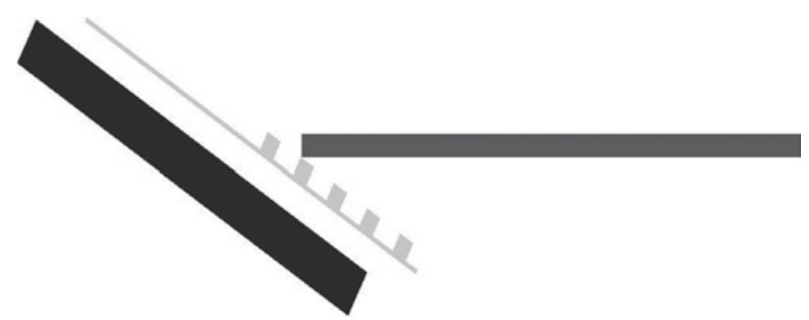

Figure 1. Initial system configuration "target - ribbed plate projectile".

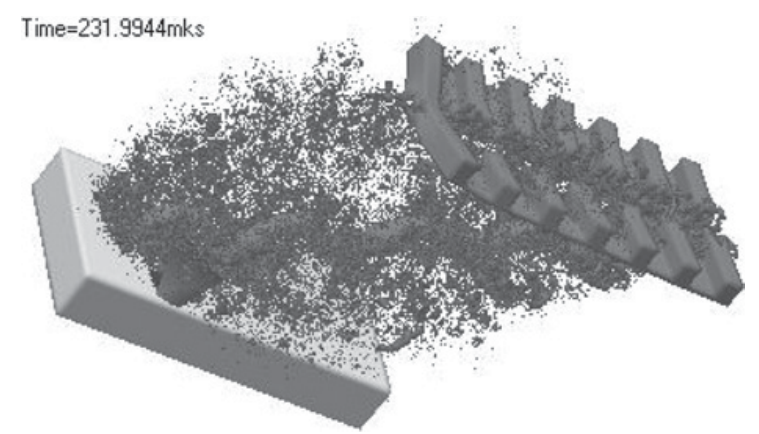

Figure 2. The three-dimensional picture interaction of projectile with ribbed plate and target.

The limiting equivalent of plastic deformation was used as a criterion of shear fracture [4] $\varepsilon^{p}=\varepsilon_{*}^{p}$. The system of Eqs. (1)-(9) is written in general form for the spatial motion of a deformable body.

The process of destruction of real materials is largely determined by internal structure of the solid, presence of heterogeneity, usually caused by different orientation of the grains in a polycrystalline material or irregularities in the composition of composite materials, the difference in micro-strength inside the grain and on inter-grain or interphase boundary. Therefore to the adequacy of the process numerically modeled with the experimental data it is necessary to bring in perturbations to physical-mechanical characteristics of the destroyed environment, i.e. to set casual distribution of the factors determining the strength properties of the material. The natural fragmentation of projectiles and target is calculated by introducing probability distribution mechanism of initial defects in the material structure to describe separated and shear cracks. The reaching its limiting value by equivalent plastic deformation is used as a criterion of fracture under intensive shear deformations.

The initial heterogeneities were modeled by that limiting equivalent plastic deformation was distributed across cells using a modified random number generator issuing a random variable obeying the chosen law of distribution.

The system of the basic equations is supplemented with appropriate initial and boundary conditions. At the initial moment all projectile points have an axial velocity $V_{0}$ with regard to its sign, the plate or rods had normal to the surface of the target velocity $\mathrm{V}_{1}$, the target state is assumed unperturbed. Boundary conditions are as follows: on borders, free from pressure, conditions are satisfied $\sigma_{n}=\tau_{n}=0$. On the contact site between bodies we

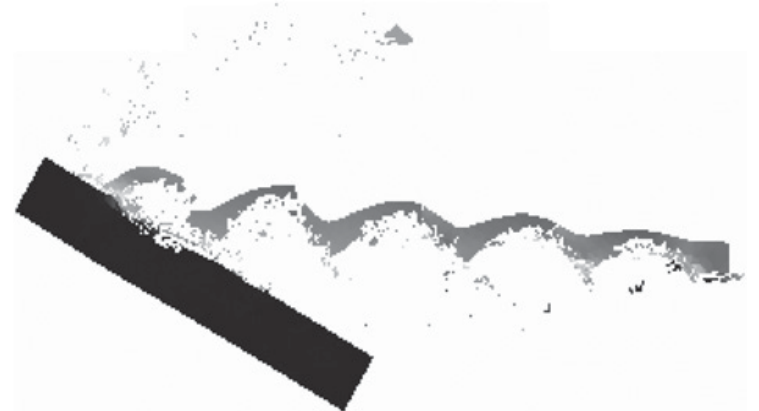

Figure 3. 2-D cross-section of three-dimensional calculated area interaction of projectile with a throwing plate and target.

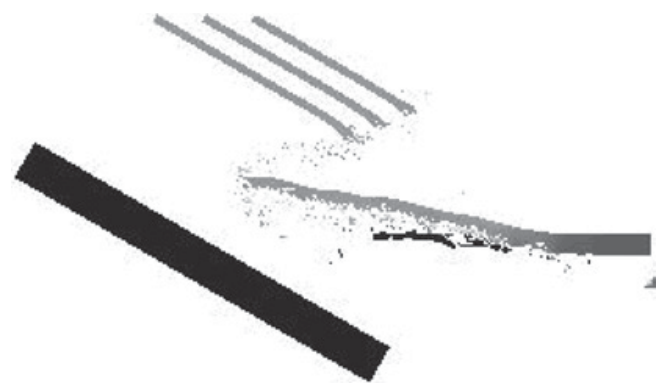

Figure 4. 2D cross section of a three- dimensional calculated area interaction of projectile with three plates.

condition ideal sliding of one material relative to another along the tangent: $\sigma_{n 1}=\sigma_{n 2}, v_{n 1}=v_{n 2}, \tau_{n 1}=\tau_{n 2}=0$, where $\sigma_{n}, \tau_{n}$-normal and tangent components of stress vector; $v_{n}$-normal component of velocity vector in a point of contact; indexes 1 and 2 refer to contacting bodies.

To calculate elastic-plastic flows one used the technique implemented on tetrahedral cells and based on joint use of Wilkins method for calculating interior points of the body and Johnson method for calculating contact interactions $[2,5,6]$. Three-dimensional area was sequentially split into tetrahedrons by means of automatic grid construction subroutines.

The most complete ideology and methodology of the probabilistic approach to the problem of solids fracture is given in $[7,8]$.

\section{The results of calculations}

Three possible impacts on high-speed elongated projectiles of tungsten alloy, approaching the target at a velocity $1500 \mathrm{~km} / \mathrm{sec}$, have been calculated. In the first case steel ribbed plate, the second case three plates of tungsten alloy, in the third case three-layer plate (middle layer - ceramics) were thrown towards the projectile.

In these cases velocity of the throwing elements was equal to $1000 \mathrm{~m} / \mathrm{sec}$ and was directed along the normal to the target. The thickness of the target was $5 \mathrm{~cm}$ and the angle of deviation from a vertical was $60^{\circ}$ (Fig. 1).

Ribbed plate is an combination of two systems - plates and rods (Figs. 1, 2).

The use the ribbed plate for protection changes the picture of its interaction with a projectile. The ribs deform the projectile to form a system of waves that can be clearly seen in Fig. 3, with their further destruction and 


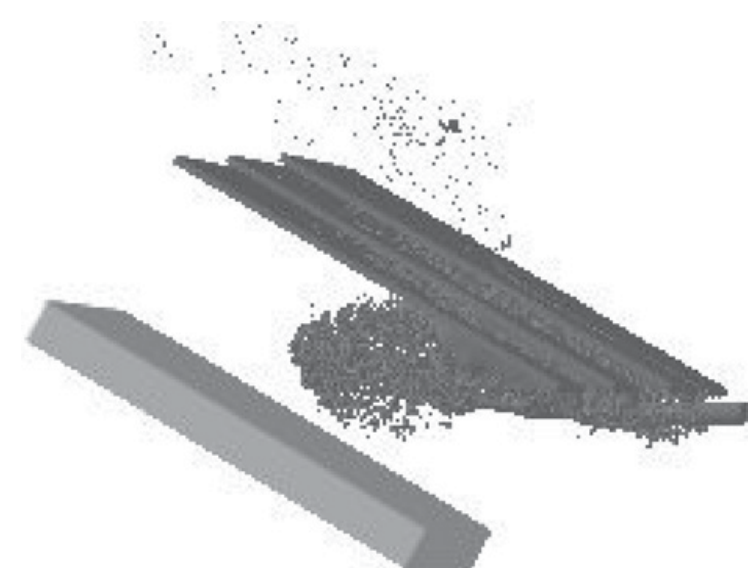

Figure 5. The three-dimensional picture of projectile interactions with three plates.

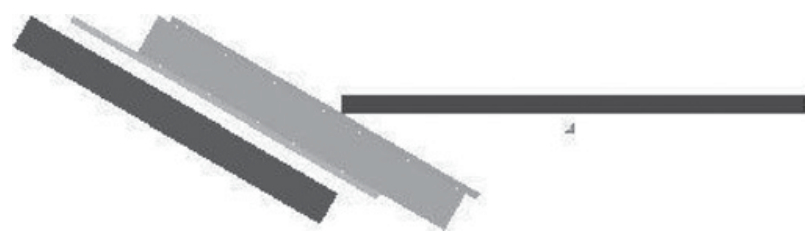

Figure 6. Interaction of projectile with the three-layer plate (middle layer - ceramics) at time $\mathrm{t}=159$ : initial system configuration "target - two plates + ceramics - projectile".

ricocheting of the obtained fragments from the surface of the target. The damages observed in the calculations are insignificant and capture only the surface layer of the material.

In this paper we have calculated two more possible scenarios of the action on approaching the target highvelocity elongated projectile made of tungsten alloy. In the first case three tungsten alloy plates were thrown towards the projectile (Fig. 4), in the second case - a three-layer plate was thrown towards the projectile (Fig. 6). A ceramic layer, equal in weight to a middle layer of the three-layer system, was placed between the tungsten layers. Physicalmechanical properties of ceramic materials are given in $[7,8]$.

In all scenarios the velocity of the thrown elements was equal to $1000 \mathrm{~m} / \mathrm{s}$ and it was directed along the normal to the target. The thickness of the target was $5 \mathrm{~cm}$ and the angle of deviation of target from vertical was $60^{\circ}$. The length of the projectile was $65.4 \mathrm{~cm}$, diameter $-2.4 \mathrm{~cm}$ and velocity $-1800 \mathrm{~m} / \mathrm{sec}$. In all cases plates of tungsten alloy had a size of $25 \mathrm{~cm}$ and $50 \mathrm{~cm}$ in width and length and $1 \mathrm{~cm}$ thickness.

When interacting with the three plates are amplified (Figs. 4,5) the destruction of the contact surface of the projectile and the degree of deviation from the direction of impact, leading eventually to the ricocheting projectile from the surface target and the preservation of its integrity.

Such character of deformation projectile and fracture can be explained by a sequence of shocks on the plates. Each subsequent plates to act already on the pre-deformed and partially destroyed part of the rod, reinforce the effect of the preceding plates.

In contrast to the above results for the three layers of tungsten alloy, the replacement of a middle layer by

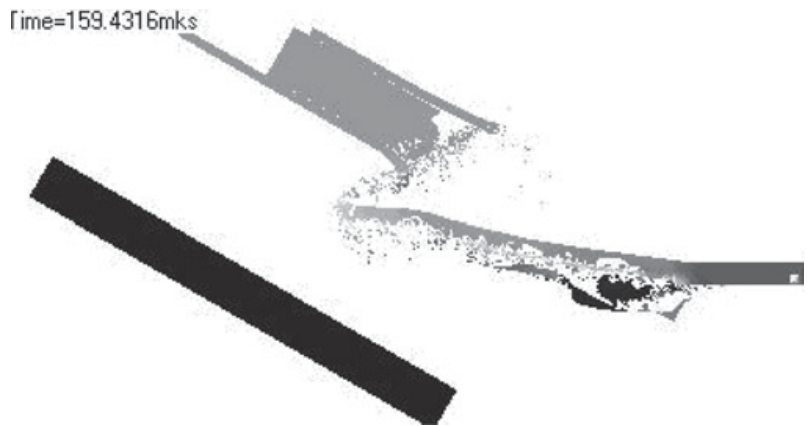

Figure 7. 2-D cross-section of three-dimensional calculated area interaction of projectile with the three-layer plate.

a thicker ceramic layer (Figs. 6, 7) prolonged the time of interaction of projectile with the system "two plates + ceramics". Impact of the ceramic layer caused more intensive destruction of the projectile in the zone of their contact and significant deviation of the projectile from the initial trajectory toward the target.

\section{Conclusions}

The aim of the present work is the numerical modeling of the processes of deformation and fracture of highspeed projectiles by elements of multilayer composite systems and different combinations of plates, as well as investigation of the impact of high-velocity projectile fragments on the main protected target. The problems were solved in three-dimensional formulation as such approach enabled us to study the processes of dynamic loading by the most adequate way to reality. In the multilayer plates included the layers of metal alloys and ceramic materials.

Calculation results showed the possibility of the proposed approaches to reduce the penetrating power of the projectile. At vigorous dynamic interaction one observes deformation and destruction of the projectiles, as well as their deviation from the line of collision with a target. Consequently the projectiles either ricochet from the target surface or deviate from the protected object and do not interact with the target. All these factors decrease the penetrating power of the projectiles and reduce probability of perforation of the protected object.

The calculations also showed that the developed $3-\mathrm{D}$ numerical method enables to simulate in complex the processes of high-velocity interaction of long projectiles with layered plates containing ceramic insets, spaced plates, rods and ribbed constructions, as well as interaction of remnants of the projectiles with protected objects in the collision along a normal and at an angle to the surface of target in a wide range of velocities and angles of impact and also to investigate the processes of fragmentation of projectile and target and a character of the fields of fragmentation.

The method takes into account probabilistic nature of the fragmentation of the colliding bodies, which leads to three dimensionality of the problem even at the impact along the normal to the target surface. Theoretical and experimental data were found to be in good agreement, which enables to use the method developed to calculate 
engineering structures subjected to impact loads and to select the most effective combinations of materials and geometrical parameters of the thrown elements.

This work was partially supported by RFBR grant No 13 -0800296-a.

\section{References}

[1] K.P. Stanjukovich (Ed), Physics of explosion (Nauka, Moscow, 1975) [in Russian]

[2] M.L. Wilkins, Fundamental Method in Hydrodynamics (Mir, Moscow, 1967) [Russian translation]
[3] M.L. Wilkins, Structural impact and crashworthiness (New York, 1984)

[4] K.N. Kreyenhagen, M.H. Wagner, J.J. Piechocki, R.L. Bjork, AIAA J. 8, 2147 (1970)

[5] G.R. Johnson, D.D. Colby, D.J. Vavrick, Int. J. Numer. Methods Engng., 14, 1865 (1979)

[6] G.R. Johnson, Trans. ASME. J. of Appl. Mech., 48, 30 (1981)

[7] A.V. Gerasimov (Ed), Theoretical and experimental researches of high-speed body interactions (Tomsk State University, Tomsk, 2007) [in Russian]

[8] A.V. Gerasimov, S.V. Pashkov, Composites: Mechanics, Computations and Applications. An International Journal, 4, 97(2013) 